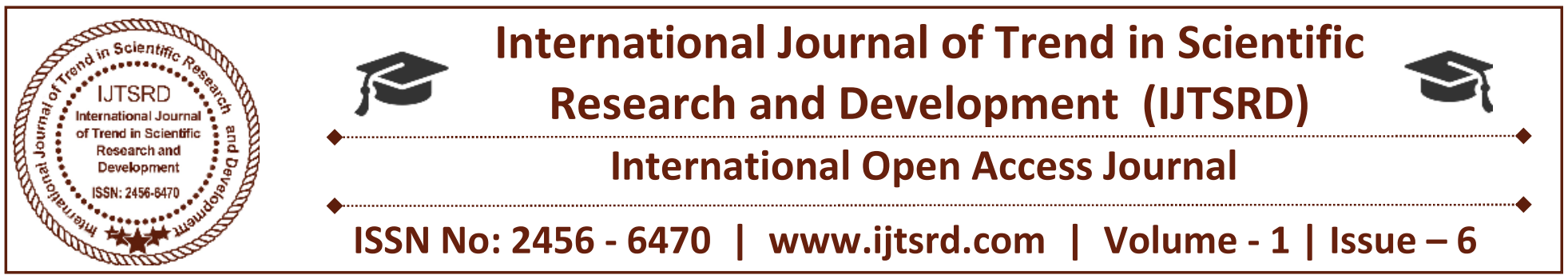

\title{
Development of the Information System of Evaluation of Multifunctional Personnel
}

\author{
Irakli Basheleishvili \\ Department of Computer Technology \\ Akaki Tsereteli State University, Kutaisi, Georgia
}

\begin{abstract}
In the work presents the issues of evaluation of multifunctional personnel, and there have been developed: the methodology for evaluation of multifunctional personnel; the evaluation stages; the evaluation scheme; formulas allowing for computing the evaluation results and for forming the matrix of functional capabilities, which represents the input indicator for the algorithm of selection and deployment of multifunctional personnel.
\end{abstract}

\section{Keywords:Multifunctional personnel, matrix of Functional capacities, Evaluation, Information System}

\section{INTRODUCTION}

Multifunctional Operator (MFO) is called a specialist with a Functional abundance who has the ability to perform one definite $f$ function in the timing of its functional capacities

$$
F_{a}=\left\{f_{e} / e \in[1, k]\right\}, k>1
$$

Compared to monofunctional operator, multifunctional operators allow us to create organizational structures of systems that have ability, in case of partial failure of any specialist, to re-adjust the whole system and facilitate its successful functioning[1,2,3].
The partial failure of the MFO is the case when he/she loses the ability to perform the function assigned to him/her but maintains the ability to

perform other functions imposed on the system based on his/her functional capabilities and may be altered to perform other functions. [1-6] It has been shown in the work that multifunctional staff has a much higher performance indicator than a system composed by mono functional specialists.

\section{Evaluation of Multifunctional Personnel}

Evaluation of multifunctional personnel consists of the following stages:

1. Registration of candidates taking part in the contest and uploading of CV into the system;

2. CV review and primary selection;

3. Different tests by using the information system;

4. An interview with a selection panel;

5. Formation of matrix of functional capabilities of multifunctional personnel.

The first stage is necessary for collecting information about personnel.

At the second stage, based on the review of the CVs of the candidates, the human resource manager carries out primary selection of those candidates, who move on to the next stage.

Those candidates, who got past the primary selection round and acquired a right to participate in the stage of testing, move on to the stage of testing. 
Testing may include different categories of tests, and these are:

1. General abilities tests.

2. Computer skills tests.

3. Professional tests.

4. Foreign language skills tests, etc.

The questions of test tasks must be prepared in accordance with the functions assigned to the system, by the appropriate competent expert or expert group.

All the contestants have to go through testing regarding all functions, and that will enable us to specify the functional capabilities (multifunctionality) of each human-operator, and to fill in the matrix of functional capabilities.

The interview process is a penultimate stage, on to which those candidates are moving, who were selected by the system as a result of primary selection and testing.

At the stage of interviewing, the selection panel must determine the maximum score, which can be collected by the candidate with account for the particular evaluation criteria. Also, there must be specified the final result of interview in the form of score, and scores must be uploaded into the information system. Once the first three evaluation stages are over, it will be possible to move on to the fourth stage, which implies determining the matrix of functional capabilities of those candidates, who passed through the evaluation stages.

All above stated stages of personnel evaluation, except for the stage of interview, have been ensured by means of the programming system, but as for the interview, as we noted earlier, it is implemented by the selection panel, whose members are to determine the final result in the form of score, which must be uploaded into the system. A score obtained by each contestant in each test category is calculated in the following way:

$$
J=\left(V_{\partial} * Q_{\partial}\right)+\left(V_{\iota} * Q_{\iota}\right)+\left(V_{\text {б }} * Q_{\text {б }}\right)
$$

where: $V$ a - the number of right answers given by the contestants to the simple questions, and $Q{ }_{\partial}$ is a score of a simple task.

$V b$ - the number of right answers given by the contestants to the questions of average complexity, and $Q \checkmark$ is a score of a task of average complexity level.

$V$ o - the number of right answers given by the contestants to the questions of high complexity, and, and $Q$ m is a score of a task of high complexity level. The formation of the matrix of functional capabilities should be carried out as follows:

weighing of scores obtained by the contestant during the test pertained to the category with a "general" status must be carried out with a score obtained by the contestant during the test pertained to the category with a "professional" status, which is calculated by using the following formula

$$
F_{i j}=\sum_{k=1}^{n} \frac{J_{k} * C_{k}}{M_{k}}+\frac{J_{j} * C_{j}}{M_{j}}+\frac{J_{3} * C_{z}}{M_{3}}
$$

where:

$F_{i j}-i$ is a score weighed with scores obtained by the contestant $j$ during the tests pertained to the categories with a "general" status.

$\sum_{k=1}^{n} \frac{J_{k} * C_{k}}{M_{k}}-\mathrm{i}-$ cumulative scores obtained by the contestant during the tests pertained to the categories with a "general" status,

where

$J_{k}$ - a score obtained by the contestant during the tests pertained to the k category;

$C_{k}$ - coefficient of a score obtained by the contestant during the tests pertained to the $\mathrm{k}$ category in the cumulative scores;

$M_{k}$ - a maximum score of the test pertained to the $\mathrm{k}$ category;

$J_{j}$ - a score obtained by the contestant $\mathrm{j}$ during the professional test $i$;

$C_{j}$ - coefficient of a score obtained during the test pertained to the $\mathrm{j}$ category in the cumulative scores;

$M_{j}$ - a maximum score of the test pertained to the $\mathrm{j}$ category;

$J_{3}$ - a score obtained by the contestant $i$ during the interview;

$C_{3}$ - coefficient of a score obtained during the interview in the cumulative scores;

$M_{3}$ - a maximum score of interview.

The coefficients $\sum_{k=1}^{n} C_{k}, C_{j}$ and $C_{3}$ in the formula (2) should satisfy the following condition:

$$
\sum_{k=1}^{n} C_{k}+C_{j}+C_{3}=1
$$


Information System of Evaluation of Multifunctional Personnel

The developed information system contains:

1. A centralized database, which represents the system's information support;

2. A set of organizational and programmingtechnical tools, which are necessary for the operation of the system, and ensure the effectiveness of the system;

3. Software system, which contains the desktop and web applications, which in turn contain the different functional modules.

The information system's generalized structural scheme is shown below in Fig. 1, which clearly illustrates how the information system's structure is organized, and which basic parts it is composed of:

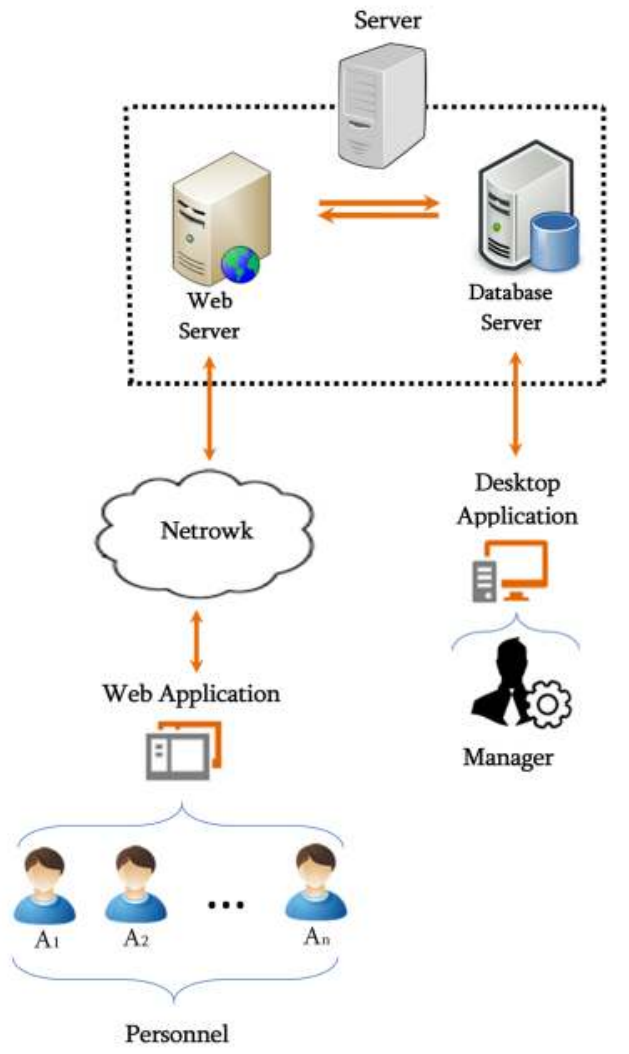

Fig. 1 Structure of the Information System

The information system's database presented in this work has been developed on the Microsoft Sql Server 2014 management system of relational databases, which represents the client-server database management system.

As can be seen from the information system's structure in Fig. 1, its software system is composed of two main modules implemented as a Desktop Application and Web Application, which in turn, contain additionally two sub-modules. The Desktop Application is intended for the automated workstation of managers, but the Web Application has been developed for the contestants (hereinafter, the websystem users), involved in the evaluation and selection processes.

\section{Desktop Application}

The Desktop Application software comprises the following functional modules:

1. registration of the organization - the module provides registration of information on the organization (used by a system) and on the organization's responsible person;

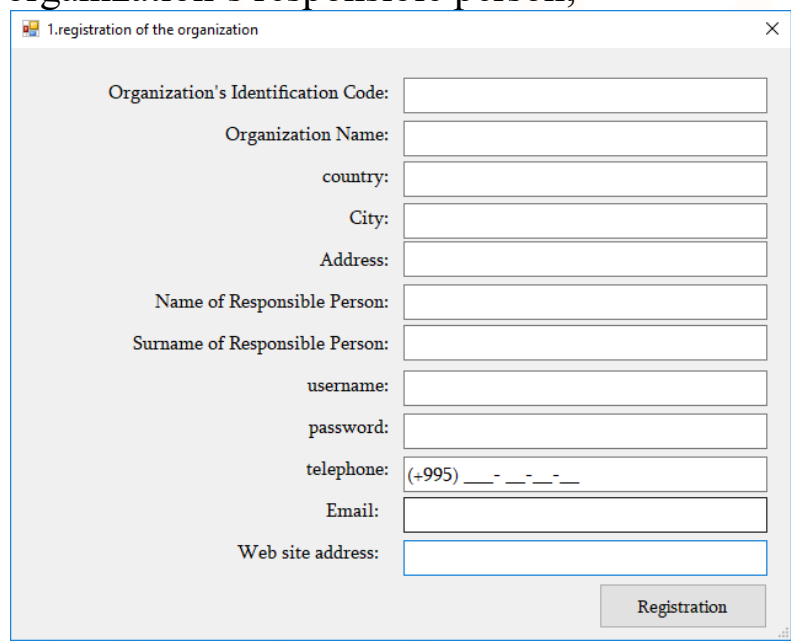

Fig. 2 registration of the organization

2. authorization - in order to $\log$ in, you have to authorize yourself. Usually, for authorization, you have to know user's name and password, which were defined at the registration stage of organization;

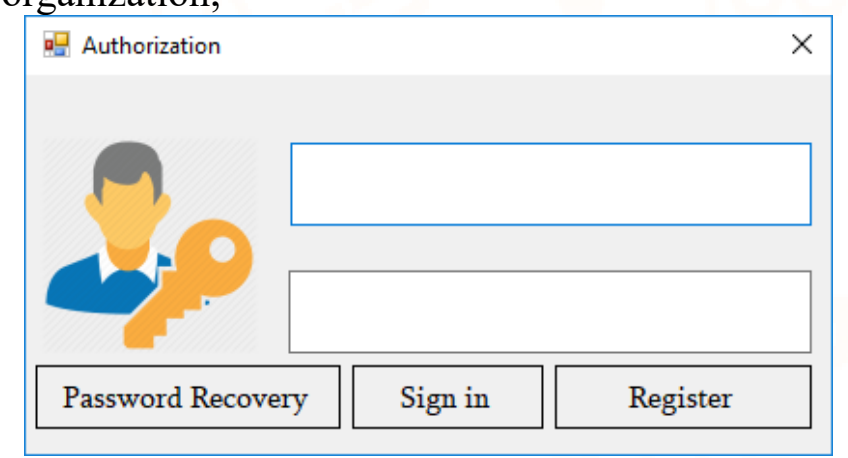

Fig. 3 authorization

3. Evaluation Category Management - the module provides the test category management: adding the category, revision (correction) of the existing version, deleting; 


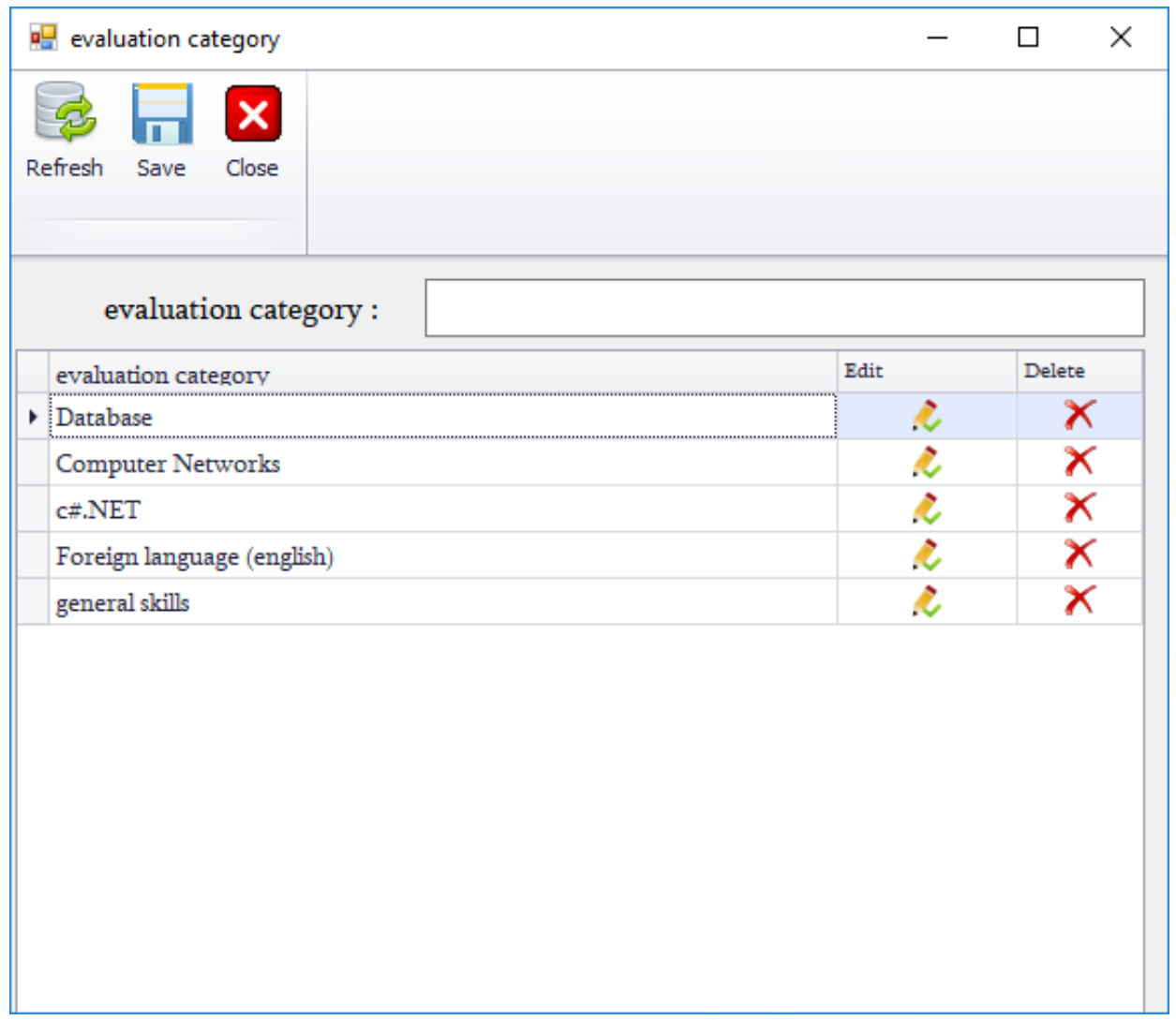

Fig. 4 evaluation category management

4. Questions Management - the module provides entering of test cases (questions and possible answers) into the database (creation of questions database), the question management module also includes the capacity of entering the questions into the database from MS Word file (*.doc or *.docx);

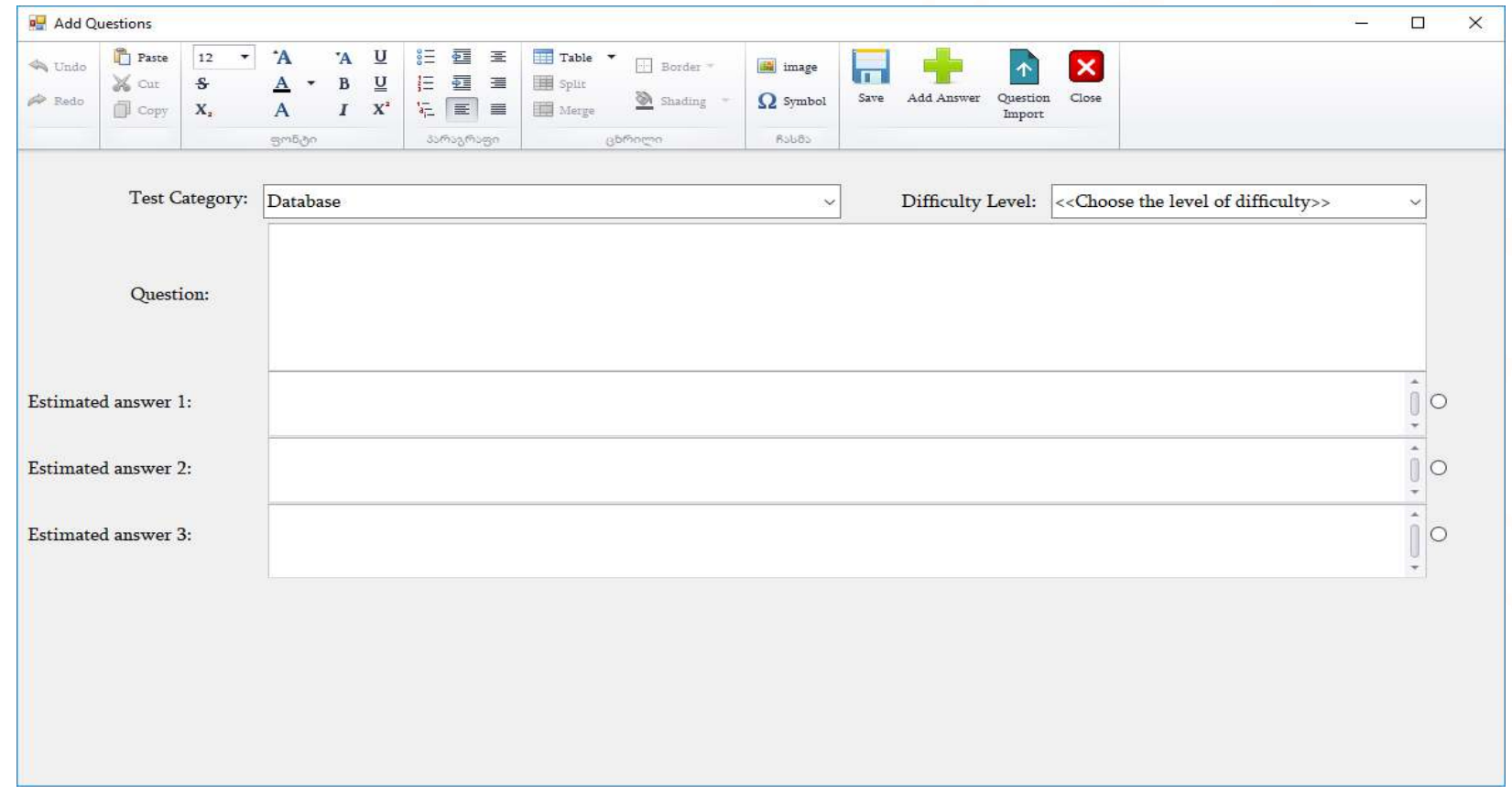

Fig. 5 Add Questions 


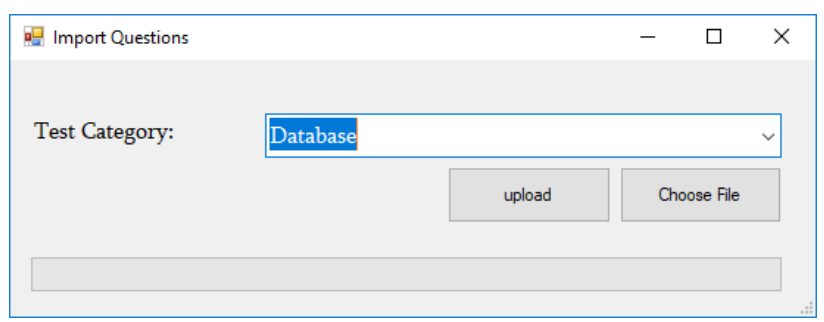

Fig. 6 Import Questions

5. Test Management - the module provides test compilation on the basis of questions entered into the database. In order to compile the test, the following parameters should be determined: test category; number of questions by the level of complexity, that is, to establish how the questions will be redistributed in a particular testing category, the number of questions with simple, average complexity and complex levels. Also, here must be determined a score for a separate question, i.e. its quantitative evaluation in accordance with the levels of complexity. Also, we can change or delete the parameters of test, which have already been compiled;

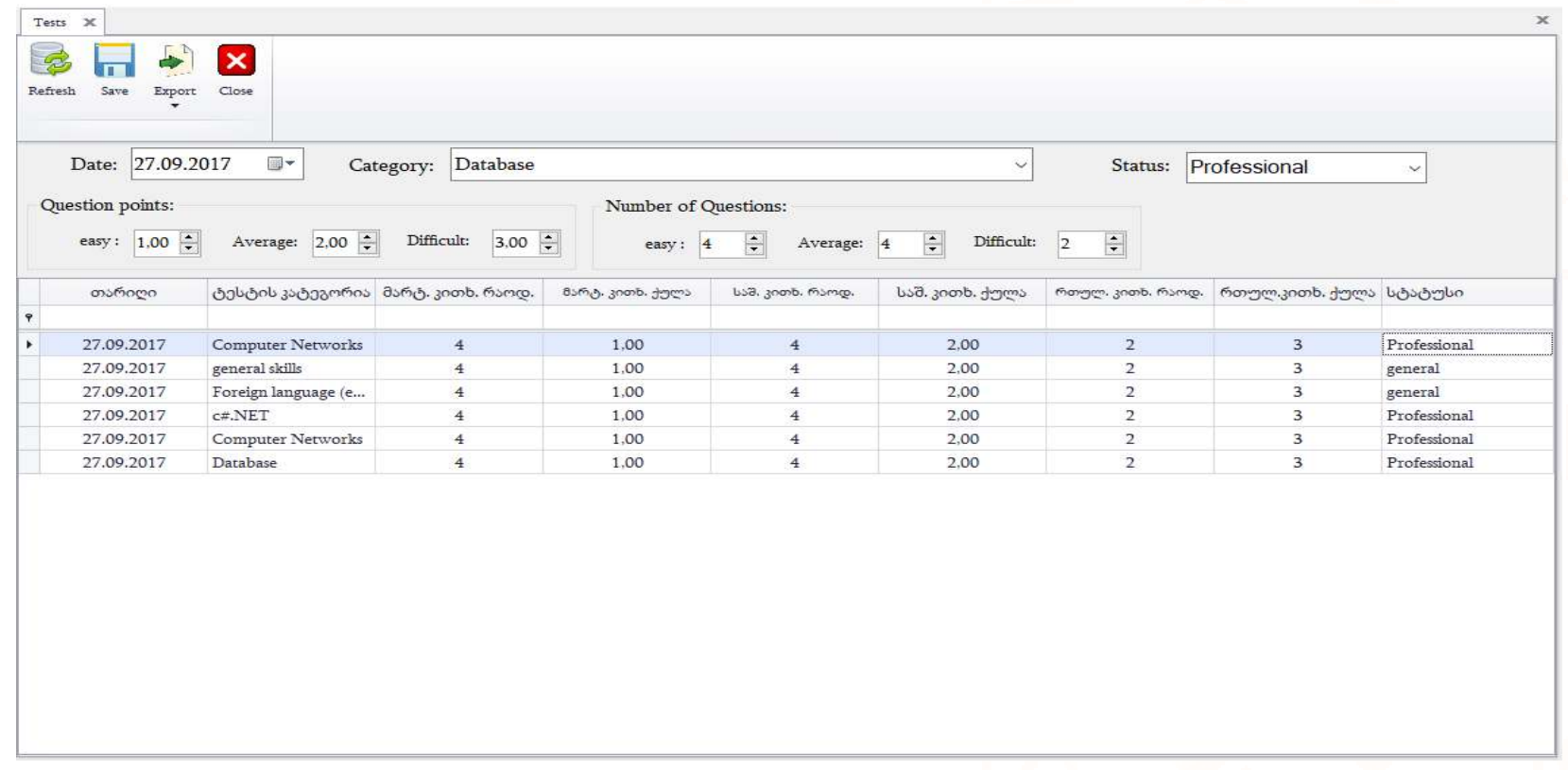

Fig. 7 Tests

6. Management For Evaluation Results - this is one of the most important modules, which allows for determining results of evaluation of tested contestants, by category of evaluation, determining the scores collected by a particular contestant in the evaluation categories, and for providing their graphical analysis. The module of management for evaluation results implies also the function of accountability for the results of interview, which means that for individual contestant passed through the stage of interview, a score formed by a selection panel as a result of interview, is entered into the database; 


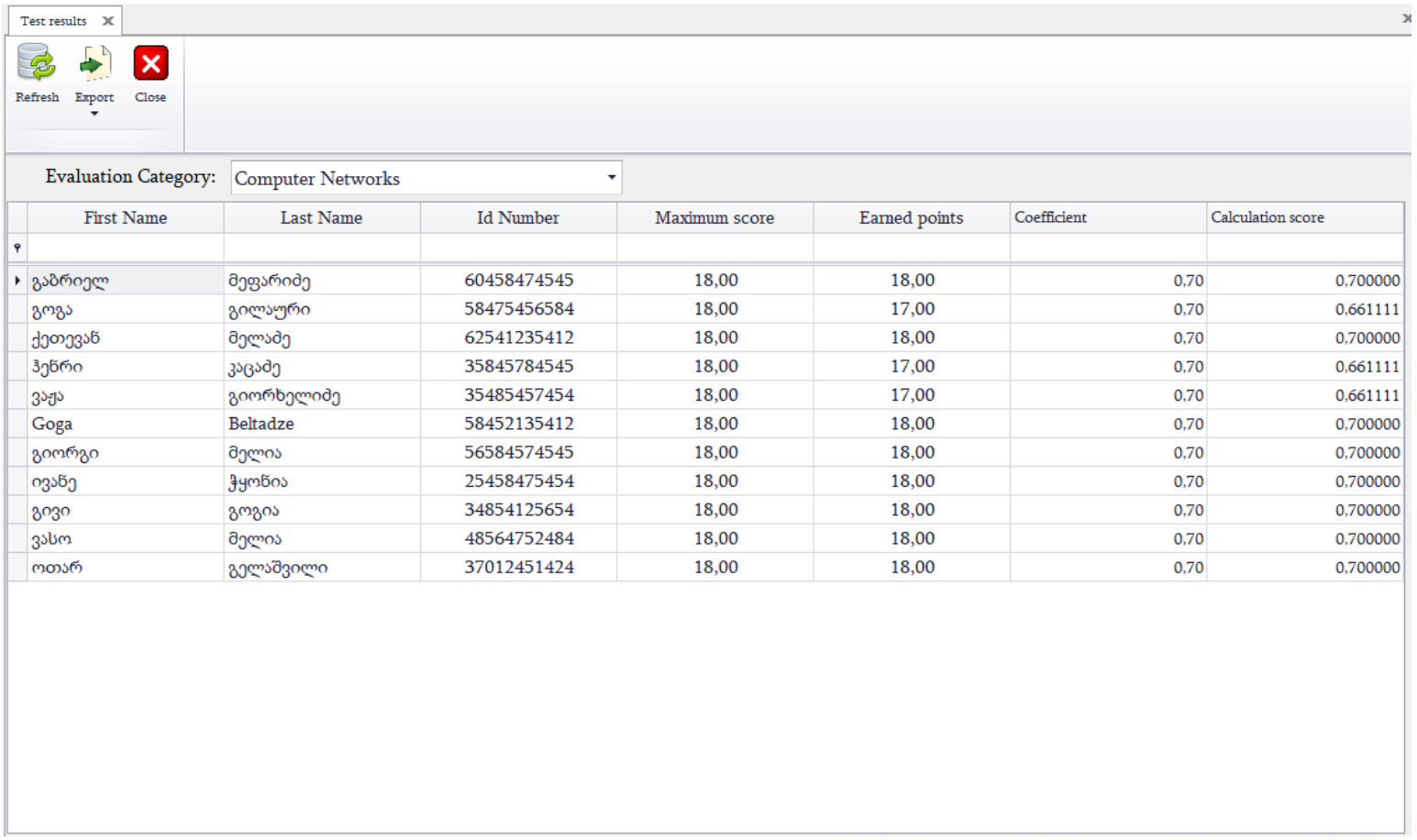

Fig. 8 Test results

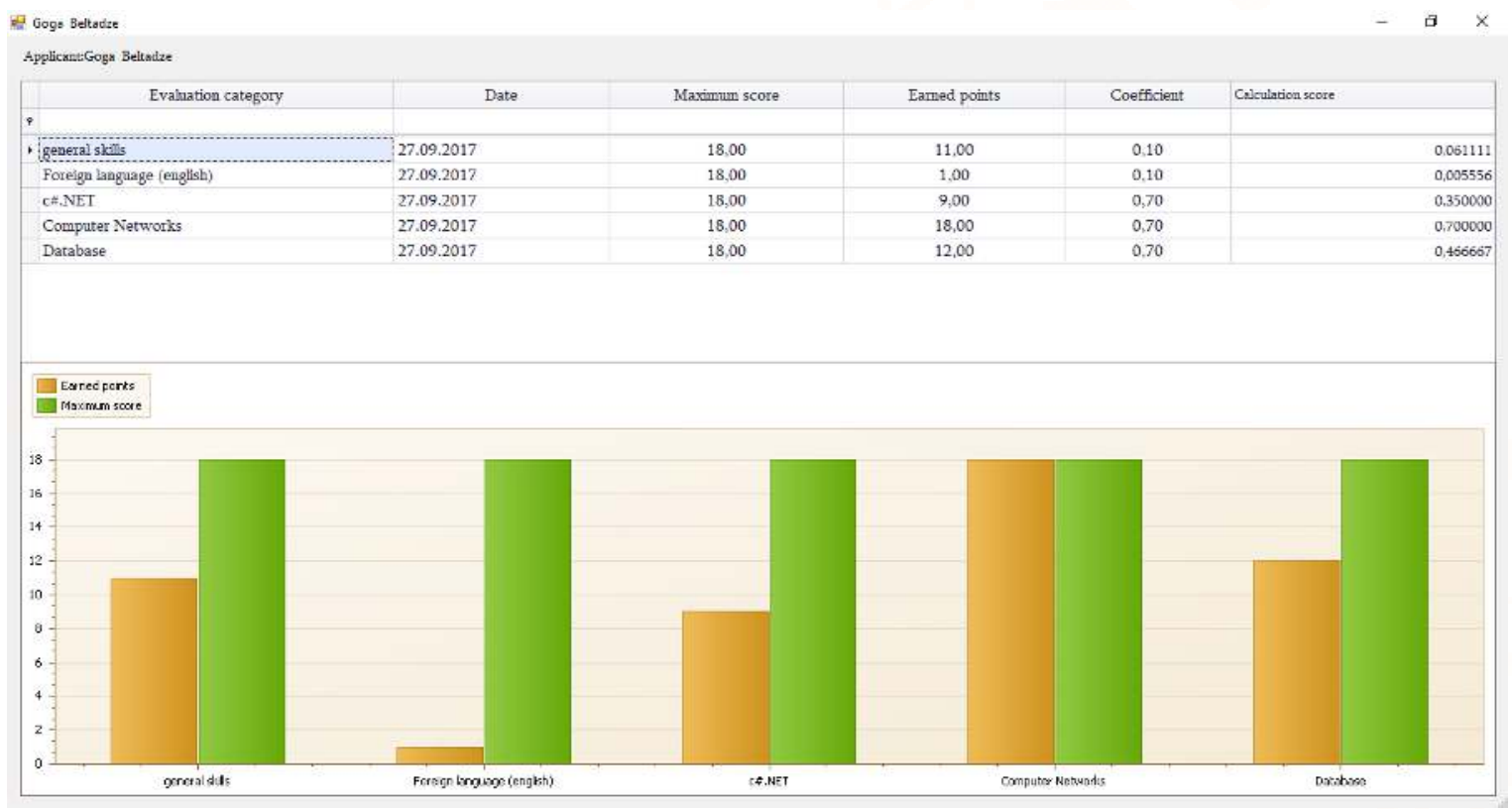

Fig. 9 Test results in detail 
International Journal of Trend in Scientific Research and Development (IJTSRD) ISSN: 2456-6470

\begin{tabular}{|l|l|l|l|l|}
\hline results of interview $\times 1$ & & & \\
\hline
\end{tabular}

Fig. 10 Results of Interview

7. Contestant Data Management - the module allows for: obtaining information (profile data, education, work experience, etc.) on all contestants registered in a system; managing access or denial of access to the next stage by means of tools specifically envisaged in the program. Where necessary, data of contestant can be deleted from the database.

\section{WEB APPLICATION}

The Web Application comprises the following functional modules:

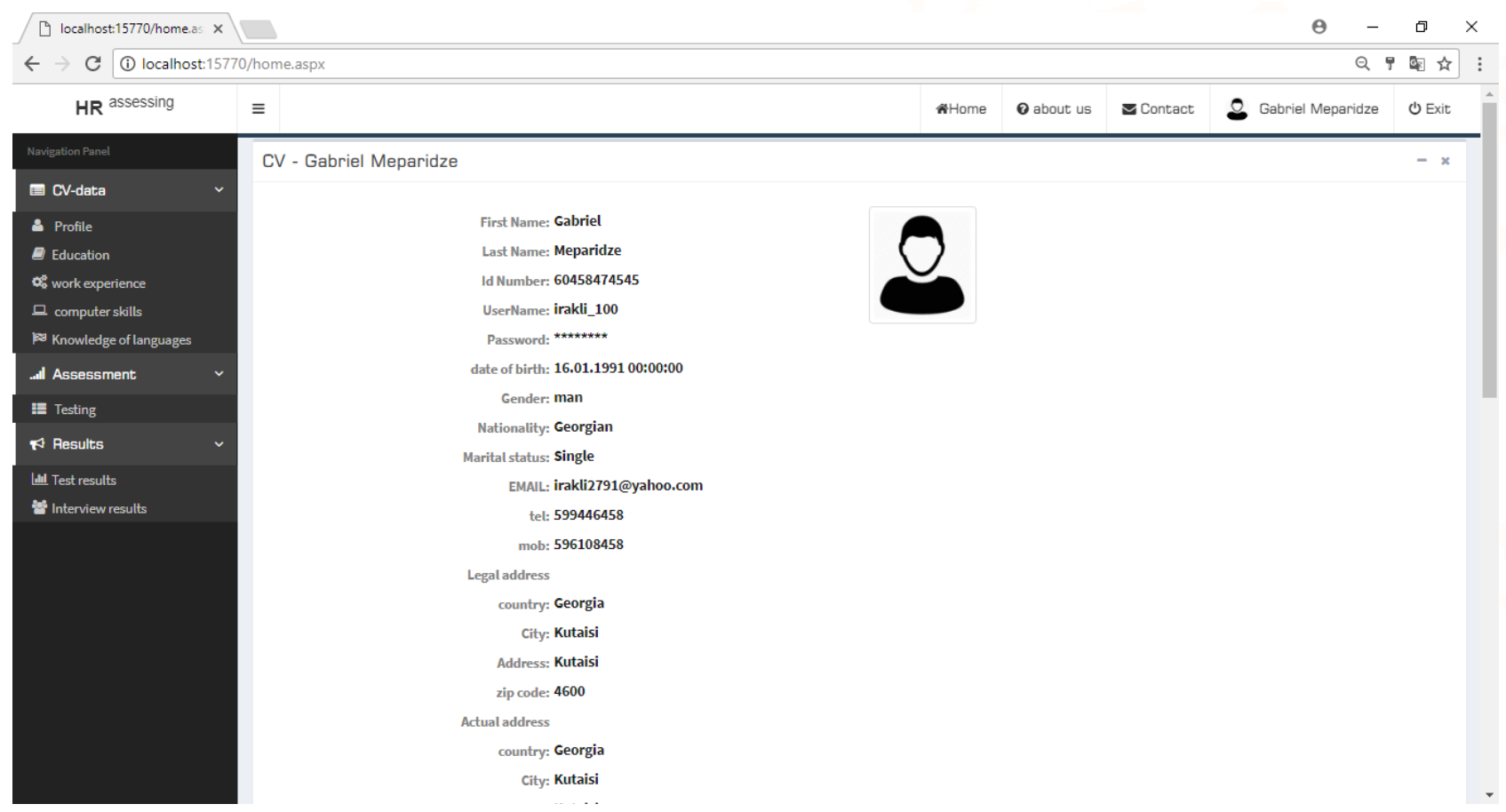

Fig. 11 web application - Main Page 
1. Registration - which provides registration of the contestant in the information system, to allow tham for creating their own accounts; The registration process is divided into four stages:

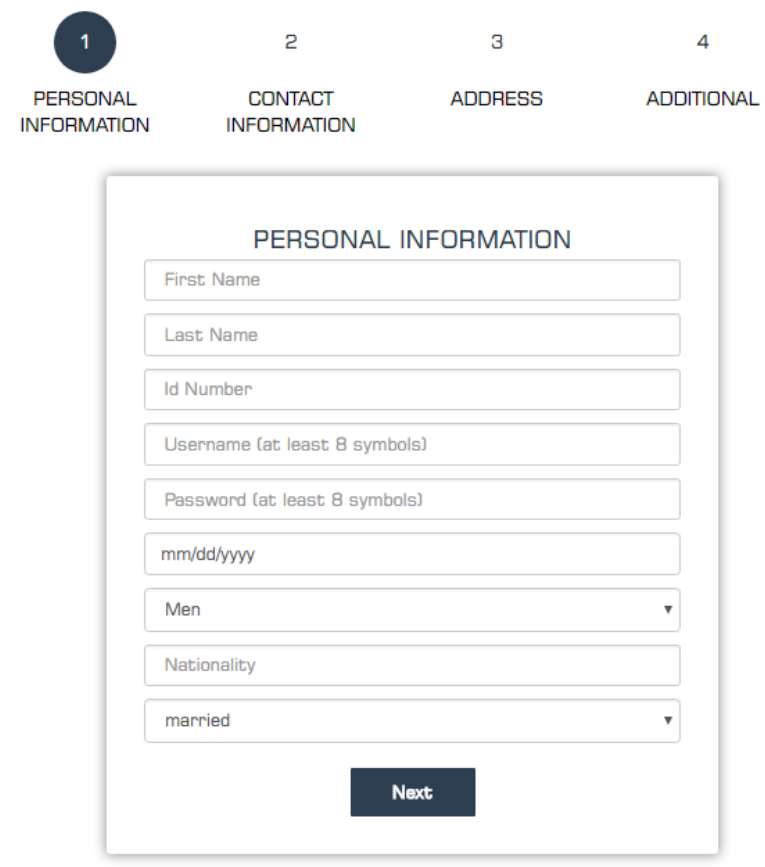

Fig. 12 Registration stage I

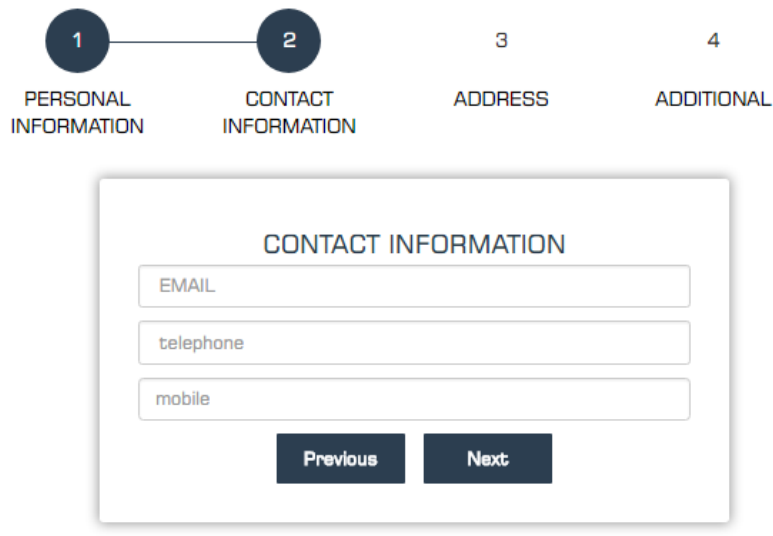

Fig. 13 Registration stage I
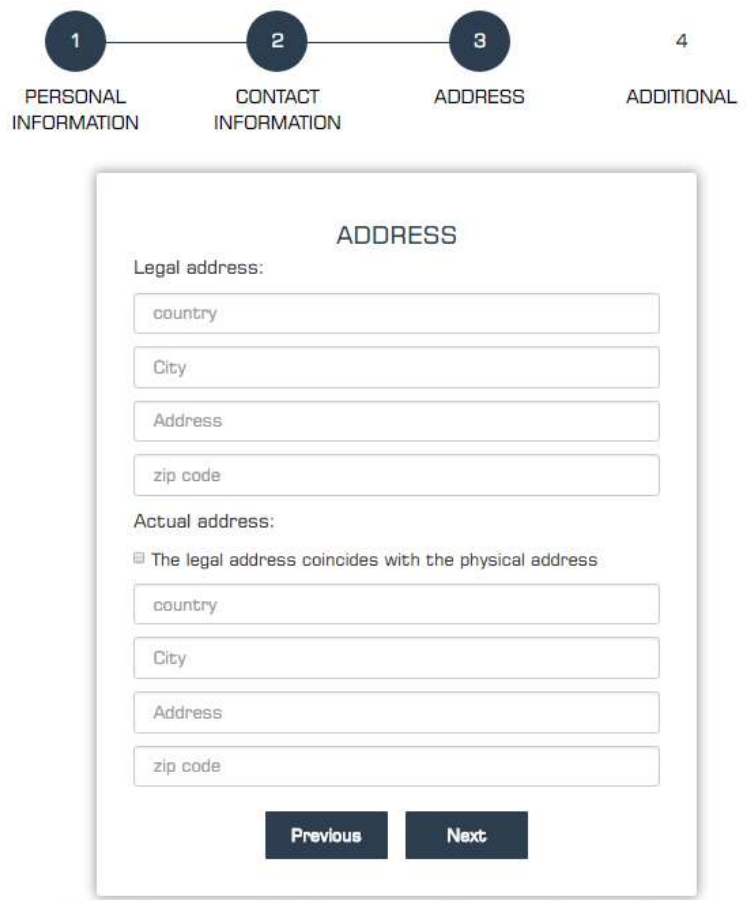

Fig. 14 Registration stage I
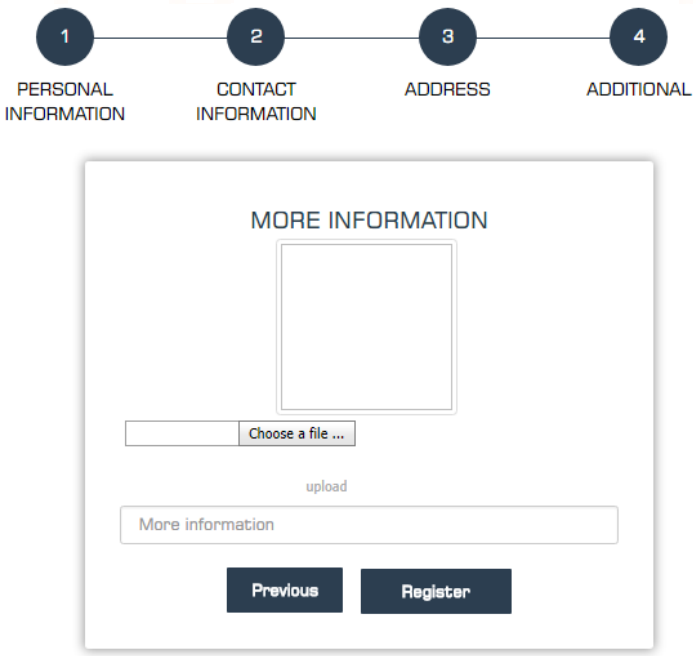

Fig. 15 Registration stage I

2. Authorization - in order to $\log$ in, the registered cotestants have to authorize themselves, for which they have to know user's name and password, which were defined by conestants at the registration stage; 
International Journal of Trend in Scientific Research and Development (IJTSRD) ISSN: 2456-6470

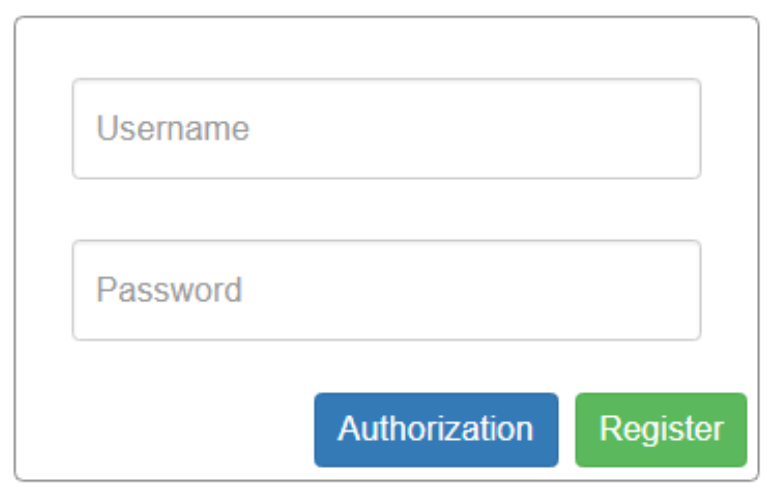

Fig. 16 authorization
3. CV - data management - this module allows the registered users for to handle easily their own data (profile data, information on education, work experience, computer and language skills, the capacity to change user's name and password in a system, and so on);

4. Evaluation - this is one of the most important modules, which allows for determining results of evaluation of tested contestants, by category of evaluation (which is defined by the manager in a system);

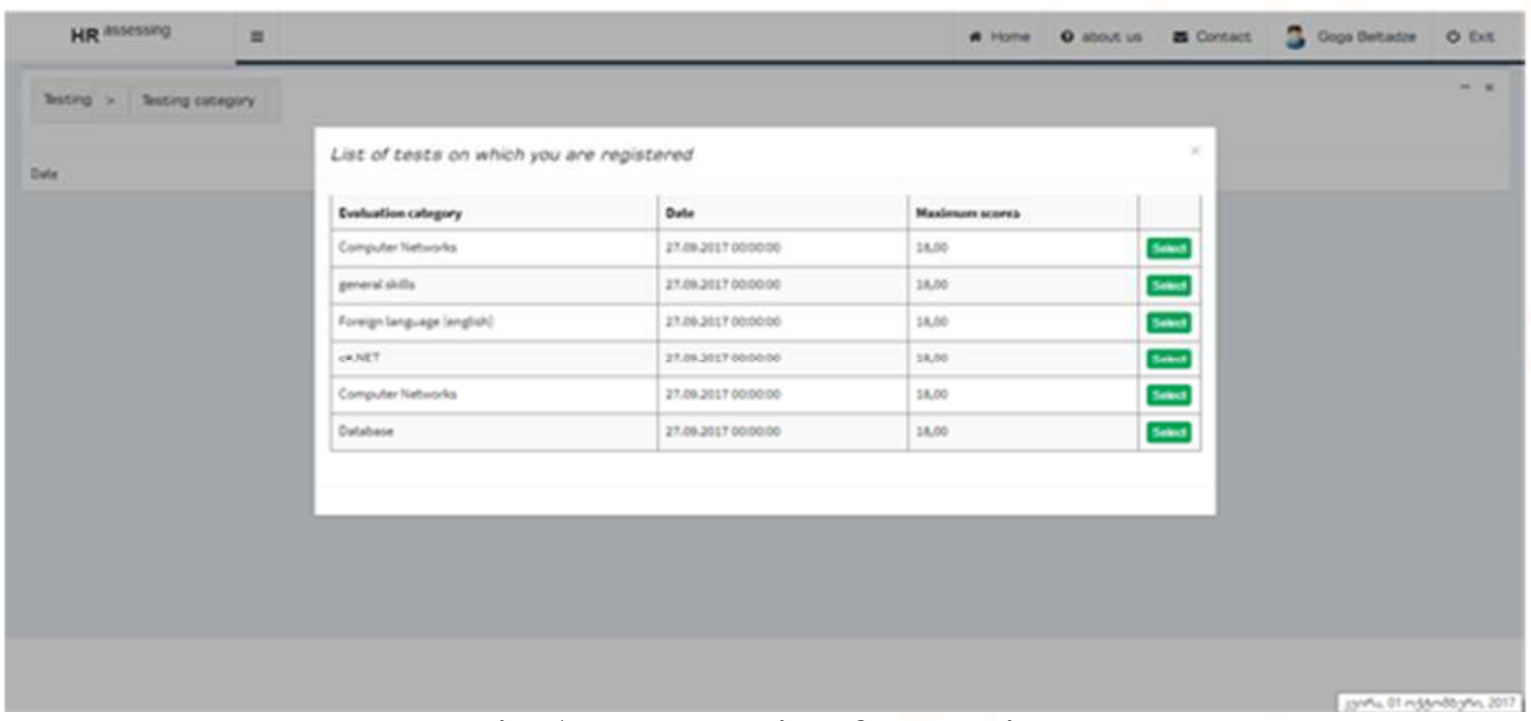

Fig. 17 category list of evaluation

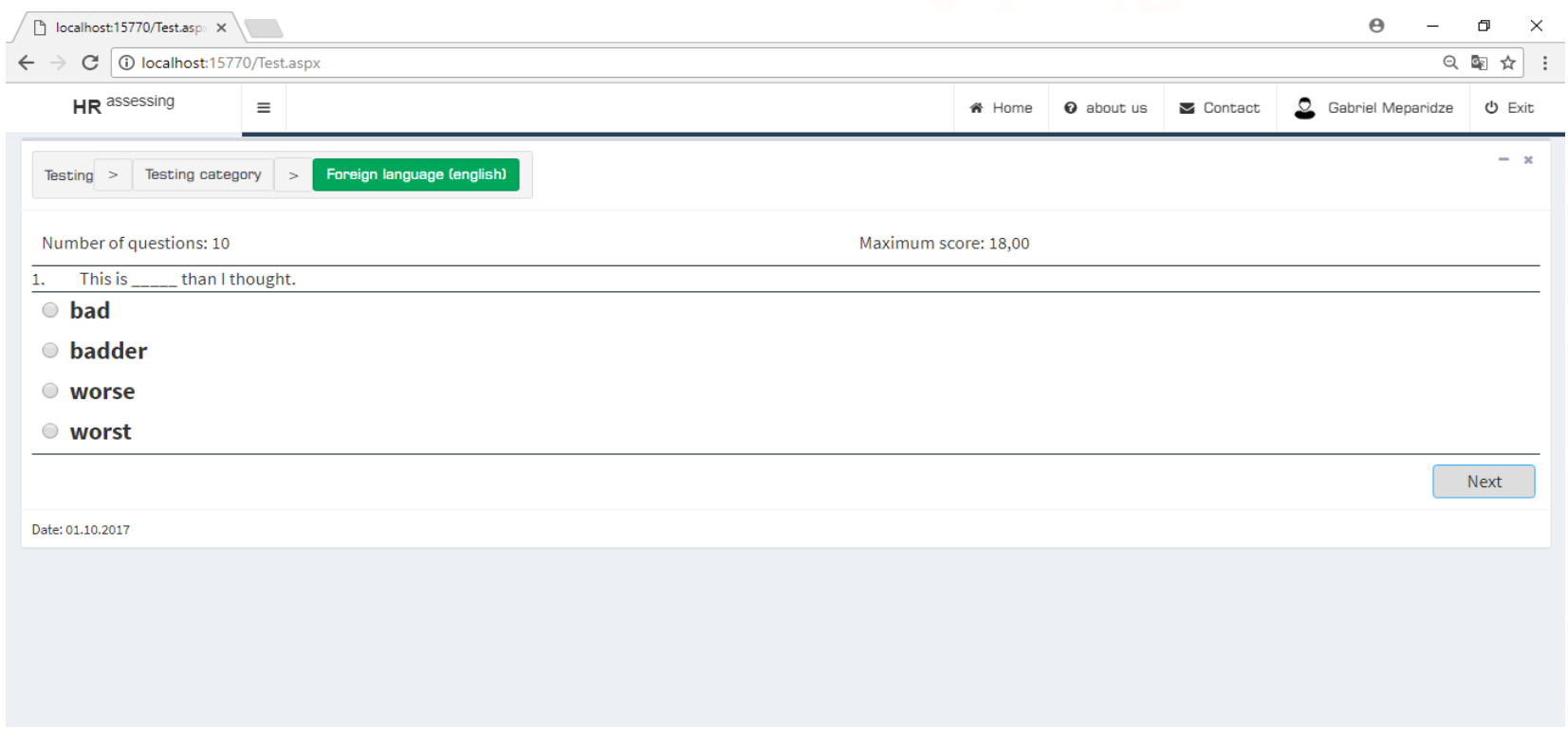

Fig. 18 Testing

8. Results - the module includes the functions, $>$ obtaining information on evaluation scores which allow the system's users for: obtained by them during the performed tests in the evaluation categories; 
International Journal of Trend in Scientific Research and Development (IJTSRD) ISSN: 2456-6470

acquainting themselves with questions used in the performed tests, in the evaluation categories, and their answers to these questions; if the user's answer was wrong, he/she has the opportunity to see which answer was correct to the appropriate question;

obtaining information on the results of interview;

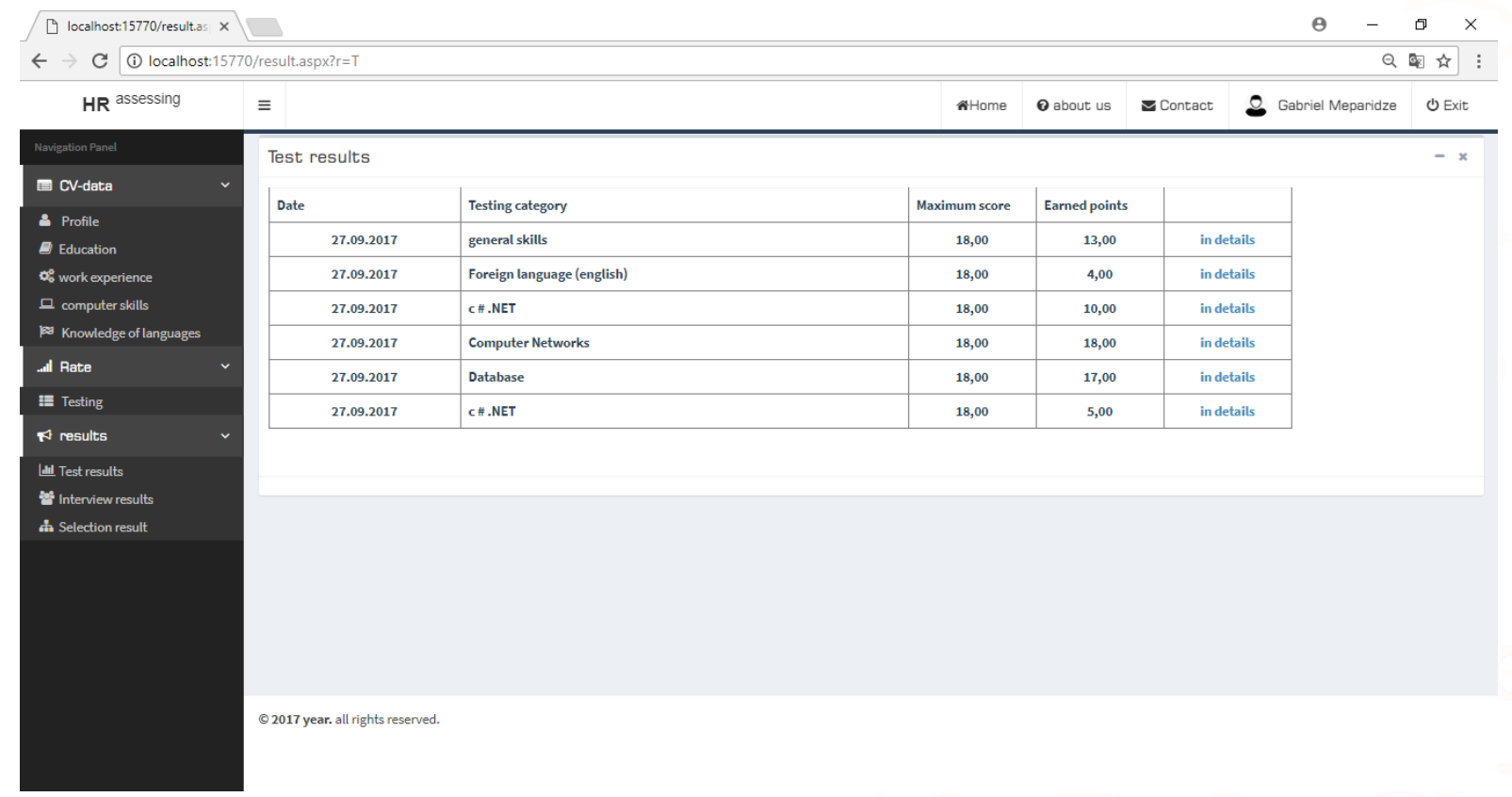

Fig. 19 Test results

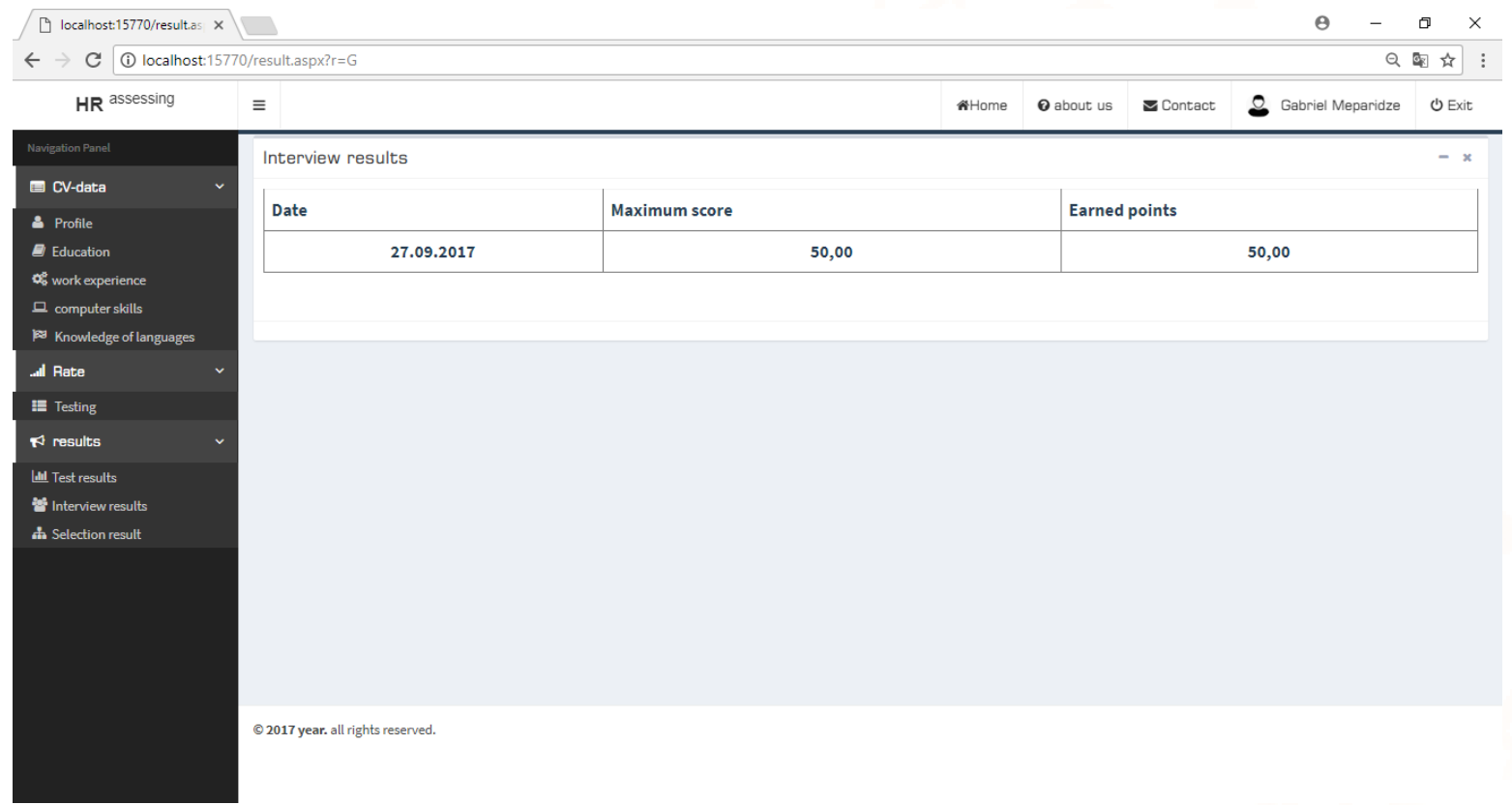

Fig. 20 Results of Interview

\section{CONCLUSION}

1. developed the methodology for evaluation of multifunctional personnel;

2. developed the multifunctional personnel evaluation scheme, for the design of the computerized system of evaluation;
3. developed the multifunctional personnel computerized system of evaluation, which provides determine a functional capability matrix based on the evaluation of multifunctional personnel. 
On the basis of the matrix of functional capabilities obtained as a result of evaluation of multifunctional personnel, we are afforded the opportunity to:

determine functional capacity of each humanoperator;

$>$ determine the indicator of reliability of the each human-operator, which is a probability of failurefree performance of a human-operator;

make optimal selection and redeployment of multifunctional personnel, by means of the algorithm developed specifically for this purpose.

\section{REFERENCES:}

[1]. I. Basheleishvili. Management Information System of Multifunctional Personnel Assessment. VIII International Scientific and Practical Conference "Internet and Society". Kutaisi, 2017. Pp. 161-165

[2]. S. Tsiramua, I. Basheleishvili. Model of Reliability of Structural Reconfiguration Multifunctional Systems. VII International Scientific and Practical Conference "Internet and Society". Kutaisi, 2015. Pp. 175-178
[3]. S.Tsiramua. Computer System of Evaluation and Management of Multi-functional Staff of Agricultural Units. Perspectives of Modern Information and Communication Systems in Agriculture, Food Production and Environmental Control (Volume B). Second European Conference of the Federation for Information Technology in Agriculture, Food and the Environment. September 27-30, 1999, Bonn, Germany. pp. 819-827.

[4].Tsiramua S.G., Kashmadze R.V. Designing of Highly Effective "Human-Computer" Systems, Based on Multifunctional Elements. Proceedings of the Fifth International Conference on HumanComputer Interaction, Orlando, Florida, 1993.

[5].Tsiramua S.G, Chikashua E.A., Tsiramua Z.G. Logical-Probabilistic Modeling, Optimization and Analysis. Computing and Computational Intelligence. Proceeding of the European Computing Conference (ECC '09). Proceeding of the 3rd International Conference on Computational Intelligence (CI '09). Tbilisi. 2009. P. 345-347 